\title{
Garlic Extract Alleviates Trastuzumab-Induced Hepatotoxicity in Rats Through Its Antioxidant, Anti-Inflammatory, and Antihyperlipidemic Effects
}

\author{
Ayman M Mousa, (1) ${ }^{1,2}$ \\ Khaled EA Soliman, $\mathbb{D}^{3,4}$ \\ Fahad Alhumaydhi, (1) ${ }^{5}$ \\ Ahmad Almatroudi, (iD) \\ Osamah Al Rugaie, (1D ${ }^{3}$ \\ Khaled S Allemailem, (D) ${ }^{1,5}$ \\ Faris Alrumaihi, (D) ${ }^{5}$ Arif \\ Khan, (D) 'Mohamad Y \\ Rezk, ${ }^{3,6}$ Mohammad \\ Aljasir, (D) ${ }^{5}$ Ameen SS \\ Alwashmi, (ID ${ }^{5}$ Faris F Aba \\ Alkhayl, ${ }^{5}$ Aqel S Albutti, ${ }^{7}$ \\ Hanan S Seleem ${ }^{3,8}$ \\ 'Department of Basic Health Sciences, \\ College of Applied Medical Sciences, Qassim \\ University, Buraydah, 5I452, Saudi Arabia; \\ ${ }^{2}$ Department of Histology and Cell Biology, \\ Faculty of Medicine, Benha University, Benha, \\ 135 I8, Egypt; ${ }^{3}$ Department of Basic Medical \\ Sciences, College of Medicine and Medical \\ Sciences, Qassim University, Unaizah, 51452 , \\ Saudi Arabia; ${ }^{4}$ Department of Forensic \\ Medicine and Clinical Toxicology, Sohag \\ Faculty of Medicine, Sohag University, Sohag, \\ 82524, Egypt; ${ }^{5}$ Department of Medical \\ Laboratories, College of Applied Medical \\ Sciences, Qassim University, Buraydah, \\ 51452, Saudi Arabia; ${ }^{6}$ Department of \\ Medical Physiology, College of Medicine, \\ Zagazig University, Al-Sharquia, 445I9, \\ Egypt; ${ }^{7}$ Department of Medical \\ Biotechnology, College of Applied Medical \\ Sciences, Qassim University, Buraydah, \\ 5I452, Saudi Arabia; ${ }^{8}$ Department of \\ Histology and Cell Biology, Faculty of \\ Medicine, Menoufia University, Shebin \\ Elkoum, Egypt
}

Correspondence: Fahad Alhumaydhi Department of Medical Laboratories, College of Applied Medical Sciences,

Qassim University, Buraydah, Saudi Arabia

Email f.alhumaydhi@qu.edu.sa
Background: Trastuzumab is a new biological drug that has been used to treat breast and gastric cancer; however, its cardiotoxicity and hepatotoxicity limit its use. Garlic has antioxidant, anti-inflammatory, antihyperlipidemic, and anticancer effects. The present study aimed to evaluate the effects of garlic on trastuzumab-induced hepatotoxicity in a rat model. Methods: Twenty rats were divided into four equal groups as vehicle control (G1), garlic $(\mathrm{G} 2)$, trastuzumab $(\mathrm{G} 3)$, and trastuzumab+garlic $(\mathrm{G} 4)$. All rats were sacrificed after eight weeks of treatment, followed by blood collection and excision of liver tissues for further analyses. The liver specimens were processed for histopathological (HP), immunohistochemical (expression of TNF- $\alpha$ and PCNA), immunofluorescent expression of Chk2 and p53, biochemical, and flow cytometry investigations to evaluate the extent of hepatocyte injury. The biochemical analysis was conducted for the activity of tissue antioxidants (GPX1, CAT, and SOD2), serum lipid profile, and liver enzymes, whereas ROS was performed by flow cytometry.

Results: The results revealed remarkable structural changes in hepatocytes of G3 with significant increases in the numbers of inflammatory cells and positive PCNA cells, area \% of collagen fibers, and immuno-expression of TNF- $\alpha$, as well as a significant reduction in the nuclear expression of Chk2. In addition, significant reductions were noticed in the antioxidant enzymes (SOD2, CAT, and GPX1) activity of G3. In contrast, the levels of lipid profile tests (triglycerides, total cholesterol, LDLC, and HDLC), liver enzymes (ALT, AST, and ALP), and ROS revealed significant increases in rats of G3. Likewise, garlic administration in G4 restored all mentioned changes to their average levels deviated by trastuzumab.

Conclusion: Based on the current results, garlic demonstrates hepatoprotective effects against trastuzumab-induced toxicity in rats. The study suggested for the first time that the coadministration of garlic with trastuzumab for treating breast or gastric cancer can augment their efficacy with minimal toxicity.

Keywords: trastuzumab, aqueous garlic extract, cancer, hepatotoxicity, oxidative stress, liver enzymes

\section{Introduction}

Breast and gastric cancer are common worldwide tumors that affect millions of patients using new biological drugs such as trastuzumab to treat these dangerous tumors. ${ }^{1}$ Trastuzumab is one of the standard safe biological adjuvant drug therapy regimens that has a significant anticancer effect. It rewards an effective regimen to treat the positive cases of human epidermal growth factor receptor 2 (HER2) breast cancer since 1998. However, trastuzumab has cardiac and hepatotoxic dysfunctions, 
limiting its use in $4 \%$ of cases. $^{2,3}$ Trastuzumab is a recombinant monoclonal HER2/neu antibody antagonist that selectively binds with high affinity to cancer cells' HER2/neu protein extracellular domain. Hence it can block their growth and stimulate the immune system to eradicate these cancer cells. ${ }^{4,5}$ The toxic effects of drugs affect the liver as it is the primary organ responsible for the biotransformation and detoxification of numerous drugs. ${ }^{6}$ Except for a few drugs that have been thoroughly investigated, such as digoxin and theophylline, it is difficult to rule out drug-induced liver injury as a complication of prescribed drugs. Several cases of hepatotoxicity have emerged in oncology, as the new therapeutic options have evolved from traditional cytotoxic effects to signal transduction inhibition, anti-angiogenesis, and immunotherapy. ${ }^{7}$ Therefore, understanding the unclear mechanisms of trastuzumab-induced hepatic injury and how to prevent it is essential. ${ }^{8}$ Herein, the alternative medicine and pharmacological attempts to protect the liver from hepatotoxicity in cancer patients are promising. However, the available data are still limited to augment the effects of these interventions. ${ }^{9}$

The use of several dietary products as hepatoprotective and antioxidants has shown immense interest in developing drugs from natural sources such as nutraceuticals. Garlic (Allium sativum) is a common herb, consumed in the foodstuff and given in the hepatic disorders since ancient times. Medicinally, garlic and its various preparations as fresh, aqueous, and alcoholic extracts have several benefits such as antioxidant, anti-inflammatory, antimicrobial, and antihyperlipidemic properties. Several studies have suggested the role of garlic as an antidiabetic, antioxidant, and immunomodulatory agent as well. The studies also showed that garlic has numerous medicinal properties due to its high concentrations of organosulfur compounds. ${ }^{11}$ Garlic offers a novel promising hepatoprotective strategy for induced hepatotoxicity in animal models, mainly through inactivation of the proapoptotic cascade and enhancing the proliferation of hepatocytes by reducing ROS formation levels. ${ }^{12}$ Evidently, it showed multiple protective effects on lead and cadmiuminduced toxicity in rats, preventing metal accumulation and biochemical alterations in the liver and kidney. ${ }^{13}$ Higher doses $(500 \mathrm{mg} / \mathrm{kg} \mathrm{BW})$ of aqueous garlic extract (AGE) significantly exhibited an ameliorative effect against the toxic oxidative injuries of lead in rats, especially on the liver, kidney, and brain. At the same time, garlic extract at different doses $(200,400,800 \mathrm{mg} / \mathrm{kg})$ ameliorated busulfaninduced testicular damages in mice with significant protection at higher doses. Although garlic possesses potent antioxidant, anti-inflammatory, antihyperlipidemic, and antimicrobial properties, its role has not yet been evaluated in trastuzumab-induced toxicity. ${ }^{10}$ In the present study, we tried to understand the role of garlic extract in trastuzumabinduced hepatotoxicity in female albino rats and its use as a supplement during breast and gastric cancer treatment.

\section{Materials and Methods} Animals and Ethical Considerations

Rats of the current study were obtained from the animal house of Qassim University (QU), KSA, kept under $23{ }^{\circ} \mathrm{C}$ on a 12-h light/dark cycle, and acclimatized at the same place for one week. Their age was ten weeks, they weighed 190-240 gm, and were fed a standard diet and water ad-libitum. The animal study protocol and procedures were accepted by the Laboratory Animal Research Ethics Committee of QU (No. mduc-2019-2-2-I-5610) and conducted following the ARRIVE guidelines and the Animal Welfare Society (University of London, UK) guidelines for the care and use of laboratory animals.

\section{Drugs and Chemicals}

Trastuzumab vials (Herceptin $150 \mathrm{mg} / \mathrm{mL}$ for injection) and aqueous garlic extract (AGE) bottles (softgel $1000 \mathrm{mg}$ ) were purchased from Genentech, Inc. (California, USA) and Puritan's pride Inc. (Oakdale, USA). Ketamine and Xylazine were purchased from Sigma (Taufkirchen, Germany). The kits of Hematoxylin and eosin stain (H\&E ab245880), Masson's trichrome stain (ab150686), HRP secondary antibody (ab6721), and DCFDA Cellular ROS assay (ab113851) were purchased from Abcam (Cambridge, UK). In addition, the primary antibodies of tumor necrosis factoralpha (TNF- $\alpha$ ABIN677318), proliferating cell nuclear antigen (PCNA, ABIN2854788), checkpoint kinase 2 (ChK2, ABIN6256554), and anti-p53 (ABIN6256312), as well as colorimetric assay ELISA kits of antioxidant enzymes (SOD2 (ABIN6959752), CAT (ABIN6976273), and GPX1 (ABIN6956204)], lipid profile tests [triglycerides (TG, ABIN772505), total cholesterol (TC, ABIN772507), lowdensity lipoprotein cholesterol (LDLC, ABIN6968342), and high-density lipoprotein cholesterol (HDLC, ABIN5708889) tests], and liver enzymes [alanine transaminase (ALT, ABIN2462887), aspartate transaminase (AST, ABIN6956178), and alkaline phosphatase (ALP, ABIN6953611)] were purchased from antibodies-online GmbH (Aachen, Germany). 


\section{Experimental Design}

The dose of garlic extract was selected after performing an in vivo oral acute toxicity assay to determine the $\mathrm{LD}_{50}$ (Supplementary Materials). Twenty adult female rats were divided equally into four groups and treated for eight weeks. We preferred the female rats for the current study as trastuzumab is mainly used to treat Her-2 expressed breast cancer, and the female rats are the well-recognized breast cancer model animal. Group 1 (G1) was treated as a control group with an intraperitoneal injection (IPI) of one $\mathrm{mL}$ of water solvent/kg once weekly. Group 2 (G2) was treated through an oral gavage tube with garlic extract $(500 \mathrm{mg} / \mathrm{kg}$ in one $\mathrm{mL}$ water solvent) once daily, ${ }^{14,15}$ group 3 (G3) was treated with IPI of trastuzumab $(10 \mathrm{mg} / \mathrm{kg}$ once weekly in one $\mathrm{mL}$ of water solvent) to induce hepatotoxicity. ${ }^{16,17}$ In contrast, group 4 (G4) was treated with IPI of trastuzumab (10 mg/ $\mathrm{kg}$ in one $\mathrm{mL}$ water solvent) once weekly and with oral garlic extract $(500 \mathrm{mg} / \mathrm{kg}$ in one $\mathrm{mL}$ water solvent) once daily. At the end of the experiment, all rats were anesthetized by IPI of $75 \mathrm{mg} / \mathrm{kg}$ ketamine and $10 \mathrm{mg} / \mathrm{kg}$ xylazine to obtain blood samples and liver specimens. ${ }^{18}$

\section{Examining Effects of Trastuzumab and Garlic on the Histological Structure of the Liver Tissues}

The excised hepatic specimens were fixed immediately in buffered formalin $10 \%$, processed to obtain four $\mu \mathrm{m} \mathrm{sec}-$ tions, and stained with $H \& E$ to evaluate the general histological structure regarding inflammatory cells infiltration (ICI) and hepatic congestion. ${ }^{19}$

\section{Evaluating Effects of Trastuzumab and Garlic on Collagen Fiber (CF) Deposition and Liver Fibrosis}

The standard Masson's trichrome staining protocol was applied to the liver tissue sections as previously described to evaluate the amount of CF deposition and the extent of fibrosis. $^{20}$

\section{Examining Effects of Trastuzumab and Garlic on TNF- $\alpha$ Immuno-Expression in the Hepatocytes}

The streptavidin-biotin peroxidase method was applied to the liver sections as previously described. ${ }^{21}$ The sections were deparaffinized, rehydrated, and incubated with the monoclonal anti-TNF- $\alpha$ or anti-PCNA antibody overnight at four ${ }^{\circ} \mathrm{C}$, secondary antibody, DAB chromogen, and Mayer's Hematoxylin (counterstain) to detect the immunoexpression of TNF- $\alpha$ or PCNA in the hepatocytes of all groups.

\section{Immunofluorescence Detection of ChK2 and p53 Proteins in Hepatocytes by the Confocal Microscopy}

The anti-ChK2 and anti-p53 antibodies were applied to the liver sections to localize sites of immunofluorescence in the hepatocytes by the confocal microscope. ${ }^{22}$

\section{Image Processing and Morphometric Study}

Ten non-overlapping fields from liver sections of each group were examined and photographed with a CMOS (TC5PRO) digital camera of a light microscope (Jinan, China) to evaluate the HP structure of hepatic tissues at the unit of advanced microscopes (QU, KSA). Quantitative statistical image analysis of 10 fields (with magnification 200X) from the liver tissues was performed by using ImageJ V1.50i (NHI/USA) software to calculate and analyze the numbers of ICs and PCNA cells /10 fields, as well as the area percentage of CF deposition (to determine the extent of hepatic fibrosis) and the immuno-expression of TNF- $\alpha$ in the hepatocytes of all groups.

\section{Effects of Trastuzumab and Garlic on Antioxidants Enzyme Assays}

According to the manufacturer's instructions, the liver tissues were excised, followed by homogenization in the assay buffer of respective assay kits to determine SOD2, CAT, and GPX1 activity.

\section{Effects of Trastuzumab and Garlic on Biochemical Analysis of Lipid Profile and Liver Enzymes}

According to the manufacturer, blood samples were collected from all rats in Eppendorf tubes and allowed to clot for $30 \mathrm{~min}$ to obtain serum (by centrifugation at $1500 \times$ $\mathrm{g}$ for $10 \mathrm{~min}$ ) and measure lipid profile and liver enzymes levels. The lipid profile parameters were TG, TC, LDLC, and (HDLC). The serum levels of liver function tests were ALT, AST, and ALP as biomarkers of hepatic injury. ${ }^{24}$ 


\section{Assay of Cellular Reactive Oxygen Species (ROS) in the Liver Tissues by Flow Cytometry}

Single-cell suspensions were acquired from the liver tissues and prepared to measure cellular ROS 2',7'dichlorofluorescein diacetate (DCFDA) in all groups by flow cytometry as described previously. ${ }^{23}$ The samples were acquired on the MACSQuant Analyzer 10 and analyzed using FlowJo software v10.7.

\section{Statistical Analysis}

The quantitative experimental data of ICs and PCNA numbers $/ 10$ fields, the area percentage of CF and TNF- $\alpha$ immunoexpression, antioxidants, lipid profile parameters, and liver function tests were calculated and statistically analyzed by One-way and Two-way ANOVA, using Prism 9. The values are expressed as mean $\pm \mathrm{SD}$ of three independent experiments. $P$-value $<0.0001$ is considered statistically significant. ${ }^{25}$

\section{Results}

\section{Garlic Effects on Trastuzumab Induced Changes in the Hepatic Tissues}

The H\& E images of hepatic tissues revealed the typical structure of hepatocytes in G1 and G2. In contrast, G3 showed noticeable hydropic changes of hepatocytes with mild ICI, and congested central veins (CCV) compared to G1, indicating the hazardous effects of trastuzumab. However, the hepatocytes of G4 showed similar features as noticed in G1 \& G2, clearly indicating the hepatoprotective effects of garlic extract supplementation with trastuzumab (Figure 1).

\section{Garlic Effects on Trastuzumab Induced Collagen Fiber (CF) Deposition Changes in the Liver}

The Masson's trichrome staining of the liver tissues exhibited mild CF deposition in the liver of G3 rats, while it was not observed in G1 and G2, indicating mild fibrotic effects of trastuzumab. Interestingly, significant changes in the CF deposition were noticed in G4, revealing a significant role of garlic extract in protecting the liver against trastuzumab fibrotic effects (Figure 2).

\section{Garlic Effects on Trastuzumab Induced TNF- $\alpha$ Immunoexpression (IE) Changes in Hepatocytes}

As depicted in Figure 3, the immunohistochemical analysis of the liver tissues revealed the absence of TNF- $\alpha$ IE in hepatocytes of G1 and G2. In contrast, hepatocytes of G3 exhibited moderate TNF- $\alpha$ IE and weak TNF- $\alpha$ IE in G4 rats, indicating the anti-inflammatory effects of garlic extract (Figure 3).

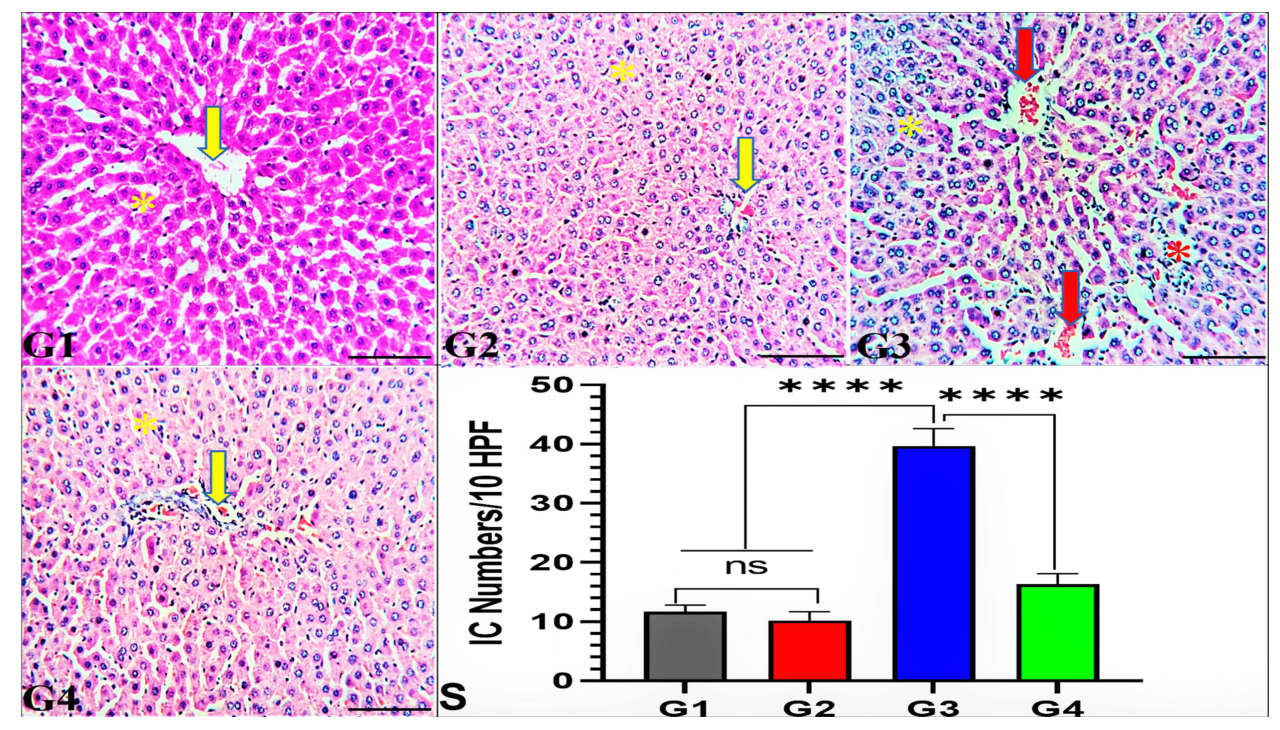

Figure I Effect of garlic extract on trastuzumab induced hepatotoxicity in rats. Liver sections from GI (control group) and G2 (garlic-treated group) show normal hepatic architecture of hepatocytes (yellow star) with normal central vein (yellow arrow). Additionally, G3 (trastuzumab-treated group) reveals hydropic changes of hepatocytes (yellow star) with focal inflammatory cells (ICs) infiltration (red star) and congested central veins (red arrow). In contrast, G4 (trastuzumab+garlic-treated group) exhibits normal features of hepatic architecture, nearly similar to GI. H\&E, 200x, bar $=100 \mu \mathrm{m}$. (S) Quantitative analysis of the ICs/I0 fields (HPF). ${ }^{\mathrm{ns}} \mathrm{No}$ significance between GI vs $\mathrm{G} 2$, *****significance difference $\mathrm{Gl}, \mathrm{G} 2$ vs $\mathrm{G} 3$, and $\mathrm{G} 3$ vs $\mathrm{G} 4(\mathrm{p}<0.00 \mathrm{I})$. 


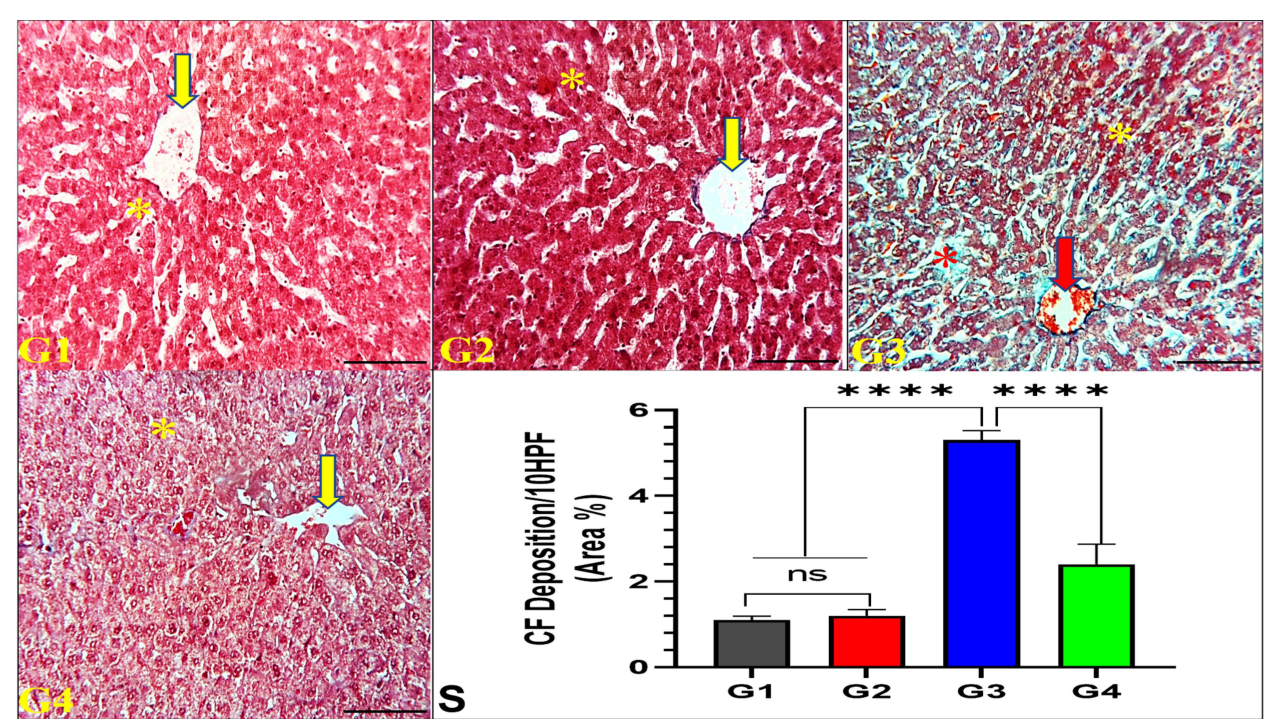

Figure 2 Effect of garlic extract on trastuzumab induced CF deposition in the liver tissues. Liver tissues from GI and G2 show typical hepatic architecture of hepatocytes (yellow star) with normal central vein (yellow arrow) and minimal CF deposition, whereas G3 reveals hydropic changes of hepatocytes (yellow star) with marked CF deposition (red star) and congested central veins (red arrow). In contrast, G4 exhibits normal features of hepatic architecture, nearly similar to GI. Masson's trichrome stain, $200 x$, bar $=100 \mu m$. (S) Quantitative analysis of CF deposition area \%/l0 HPF. ns No significance between GI vs G2, ****Significance difference GI, G2 vs G3, and G3 vs G4 ( $p<0.00 \mathrm{I}$ ).

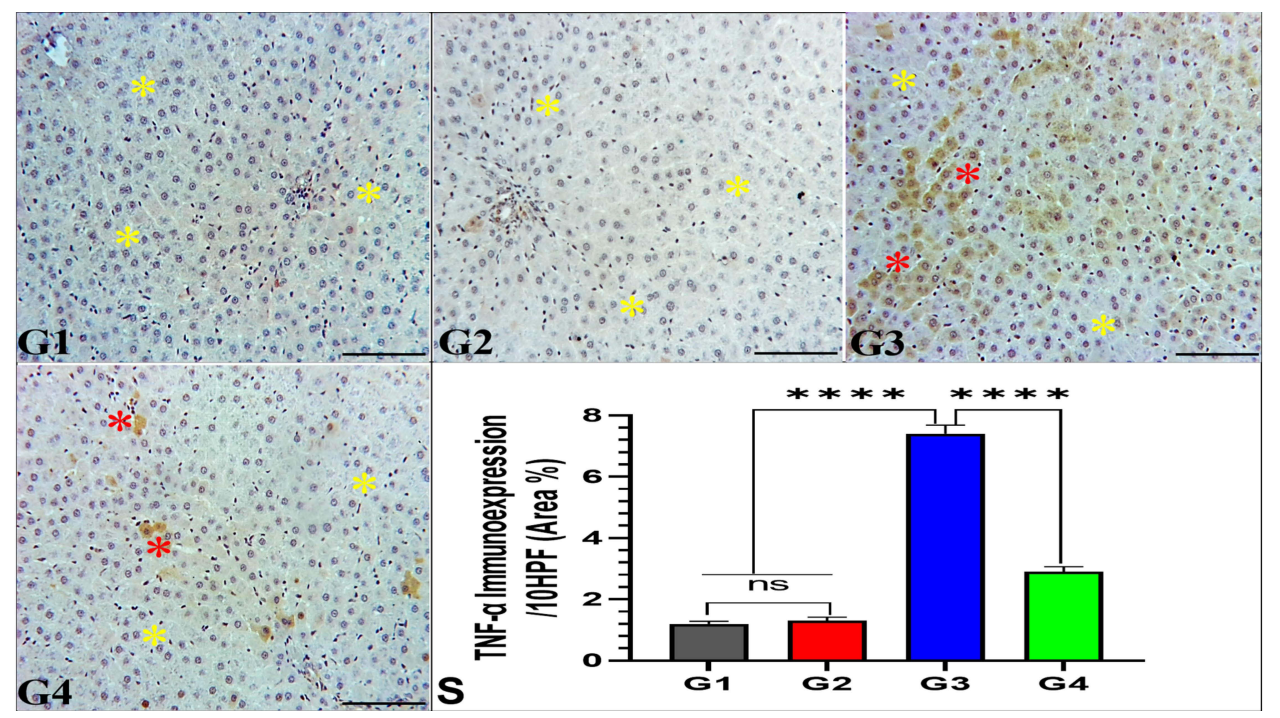

Figure 3 Effect of garlic extract on trastuzumab-induced TNF- $\alpha$ changes in the liver tissues. Liver tissues of GI and G2 show normal hepatic architecture of hepatocytes without TNF- $\alpha$ immunoexpression (IE) at the yellow stars, while the liver tissues of G3 reveal focal areas of marked TNF- $\alpha$ IE (red stars) in-between normal hepatocytes without TNF- $\alpha$ IE (yellow stars). In contrast, G4 exhibits normal hepatic architecture without TNF- $\alpha$ IE (yellow stars) and focal areas of minimal TNF- $\alpha$ IE (red stars). TNF- $\alpha$ immunostaining, 200x, bar $=100 \mu \mathrm{m}$. (S) Quantitative analysis of TNF- $\alpha \mathrm{IE}$ area \%/I0 HPF. ${ }^{\mathrm{ns}}$ No significance between GI vs G2, *****Significance difference GI, G2 vs G3, and G3 vs G4 ( $\mathrm{P}<0.00 \mathrm{I}$ ).

\section{Garlic Effects on Trastuzumab Induced PCNA IE Changes in the Hepatocytes}

The expression of PCNA showed a significant reduction in the liver of G3 rats compared to G1 and G2, indicating the anti-mitotic activity of trastuzumab in G3. In contrast, a significant increase of PCNA in G4 compared to G3 indicated the mitotic effects of garlic extract supplemented with trastuzumab on hepatocytes of G4 (Figure 4).

\section{Garlic Effects on Trastuzumab-Induced Immunofluorescence Changes of ChK2 and p53 in the Hepatocytes by Confocal Microscopy}

The immunofluorescence analysis of ChK2 and p53 proteins were conducted under the confocal microscope, which revealed a significant reduction in the expression of Chk2 in the nuclei of G3 due to the stress generated by trastuzumab in G3. However, the nuclear localization of 


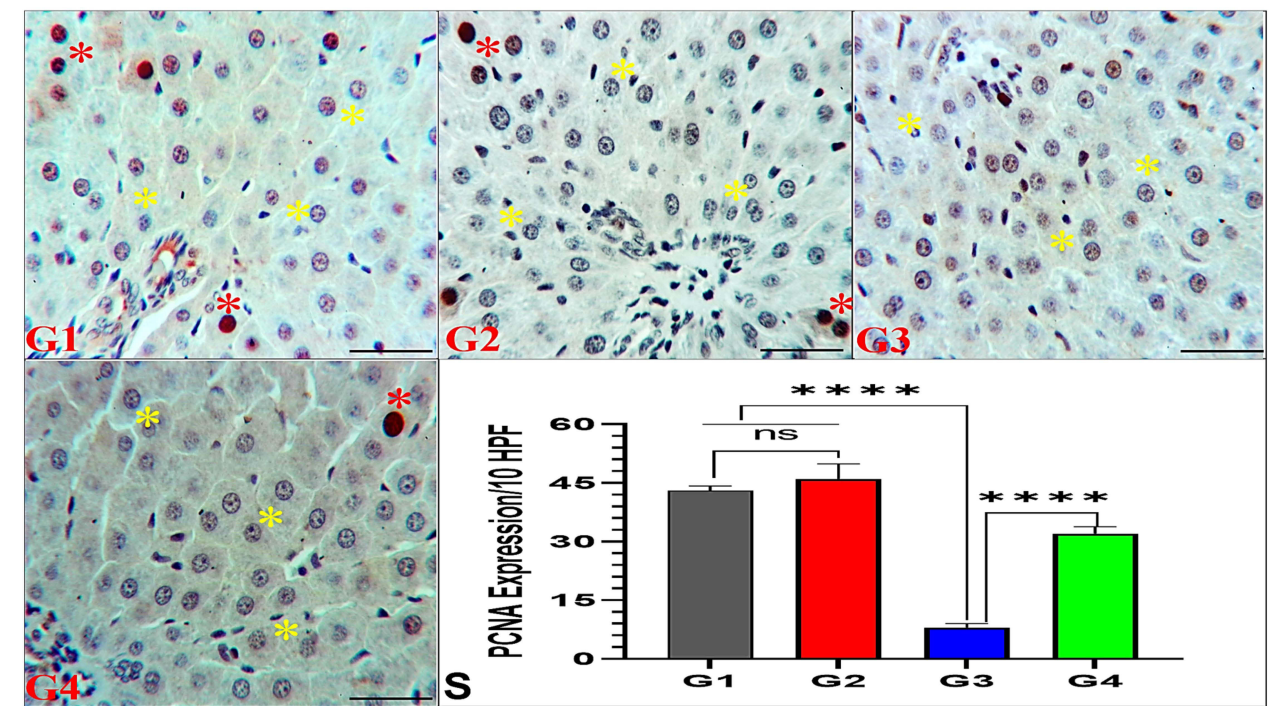

Figure 4 Effect of garlic extract on trastuzumab-induced PCNA expression in the liver tissues. Liver tissues of GI and G2 show normal hepatic architecture of hepatocytes (yellow stars) and some hepatocytes with obvious PCNA IE of mitotic brown nuclei (red stars). In contrast, the liver tissues of G3 shows hepatocytes without PCNA IE (yellow stars) and G4 exhibits normal hepatic architecture (yellow stars) with PCNA IE of brown nuclei in some hepatocytes (red stars). PCNA immunostaining, 200x, bar $=100 \mu \mathrm{m}$. (S) Quantitative analysis of PCNA IE area \%/I0 HPF. ns No significance between GI vs G2, ****Significance difference GI, G2 vs $\mathrm{G} 3$, and $\mathrm{G} 3$ vs $\mathrm{G} 4(\mathrm{p}<0.00 \mathrm{I})$.

Chk2 was restored by garlic extract in G4. Interestingly, no change in the expression of $\mathrm{p} 53$ was observed in any treated groups, indicating the independent stress of $\mathrm{p} 53$ (Figure 5).

\section{Garlic Effects on Trastuzumab Induced Changes of Antioxidants Enzymes in the Liver Tissues}

The data showed significant reductions of SOD2, CAT, and GPX1 levels in the liver tissues of G3 compared to G1 \& G2. Noticeably, a significant increase was observed in $\mathrm{G} 4$, indicating the antioxidant effects of garlic extract on the liver tissues (Figure 6).

\section{Garlic Effects on Trastuzumab Induced Changes of the Serum Lipid Profile}

Figure 7 revealed significant elevations of TG, TC, and LDLC and reduction of HDLC in G3 rats, indicating the hyperlipidemic effects of trastuzumab compared to G1 \& G2. In contrast, coadministration of garlic extract with trastuzumab has shown significant restoration of these changes, demonstrating the antihyperlipidemic effects of garlic extract on G4 rats (Figure 7).

\section{Garlic Effects on Trastuzumab Induced Changes of the Serum Liver Function Tests (LFTs)}

As depicted in Figure 8, significant elevations in the ALT, AST, and ALP serum levels were measured in G3 after trastuzumab administration. In contrast, garlic administration in G4 resulted in remarkable reductions in these levels towards the average values.

\section{Garlic Effects on Trastuzumab Induced Changes of Cellular ROS Levels by Flow Cytometry}

The detected data by mean fluorescence index (MFI) of DCFDA revealed a significant increase in the cellular ROS analysis of G3 hepatocytes (14433.3 MFI). The MFI in G1 and G2 was 2716.7 and 2506.9 MFI, respectively, while it was 7226.5 MFI in G4 rats after garlic supplementation. The ROS changes validated the antioxidant effects of garlic extract on the liver tissues (Figure 9).

\section{Discussion}

Generally, trastuzumab administration once a week is considered a highly safe regimen, which increases the overall survival rate in patients with HER-2 positive breast cancer. ${ }^{26}$ Nevertheless, few cases of hepatotoxicity have been reported, and its toxicity is not related to the dose or 

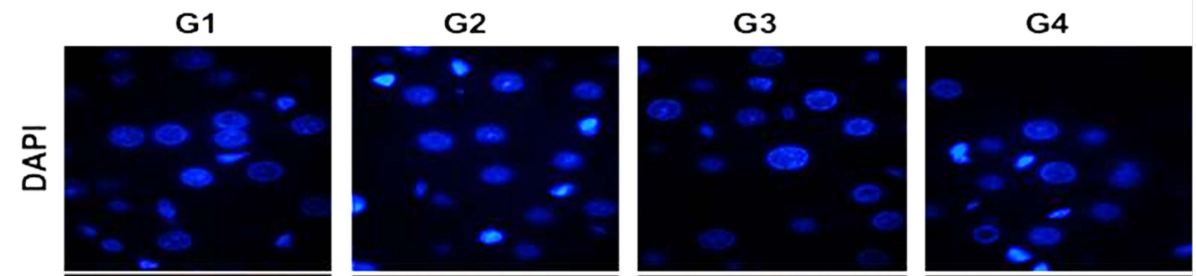

\section{S}
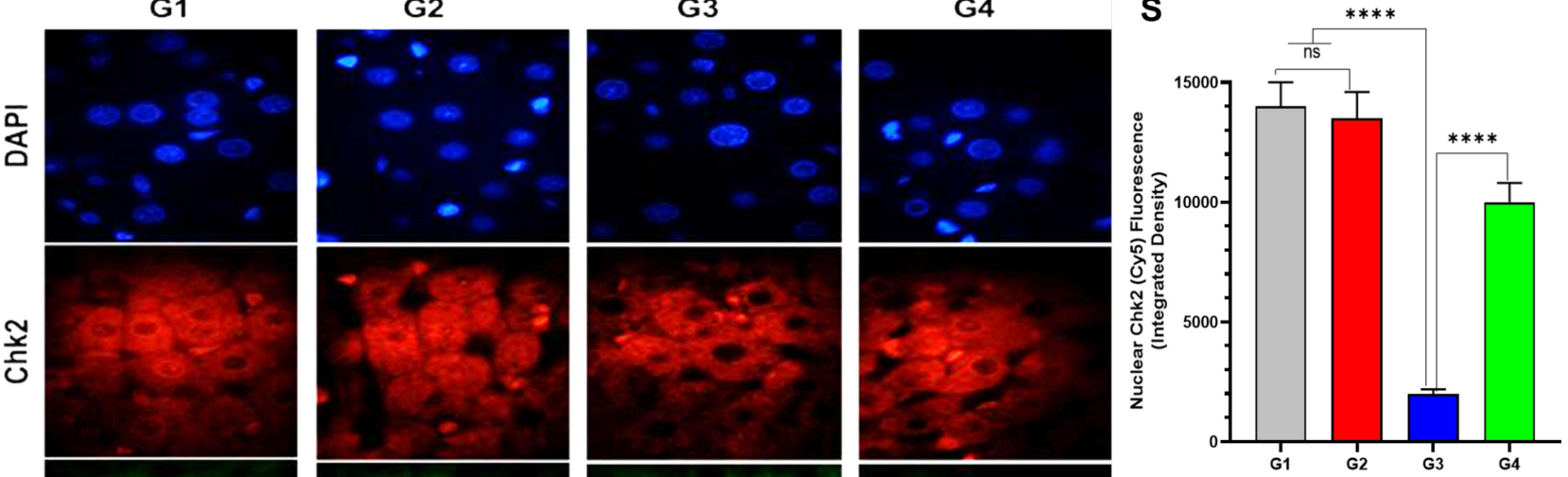

ก
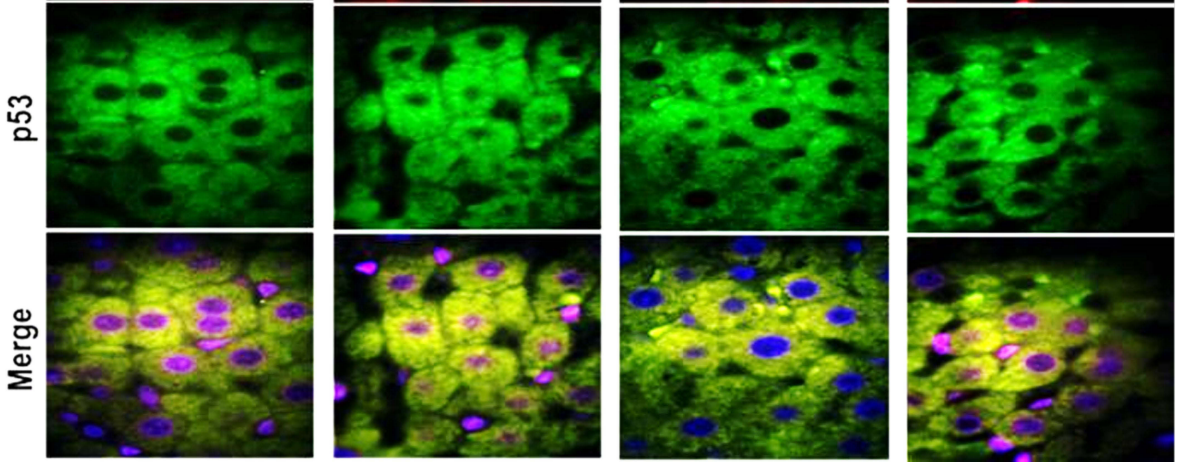

Figure 5 Effect of garlic extract on trastuzumab-induced Chk2 and p53 expression in the liver tissues. Immunofluorescence images from the liver tissues representing DAPI (blue staining), ChK2 (red staining), p53 (green staining), and their co-localization (merged images). (S) Quantitative analysis of the Chk2 nuclear expression as fluorescence (integrated density). ${ }^{\text {ns }}$ No significance between GI vs G2, ****Significance difference GI, G2 vs G3, and G3 vs G4 ( $p<0.001$ ).

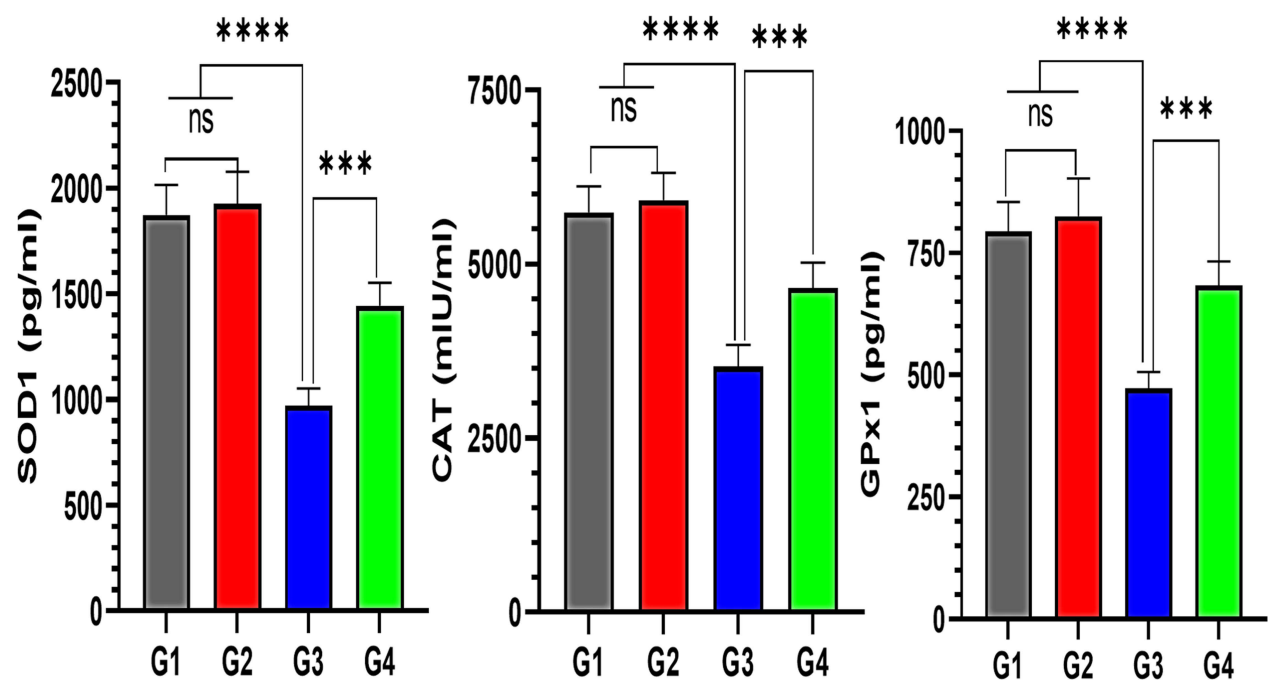

Figure 6 Quantitative analysis of garlic extract effects on trastuzumab-induced changes in the antioxidant enzymes levels of the liver tissues. G3 reveals significant decreases in the levels of (A) superoxide dismutase (SOD2), (B) catalase (CAT), and (C) glutathione peroxidase (GPXI) compared to GI, G2, and G4 in the liver tissues. ${ }^{\text {ns }} \mathrm{No}$ significance between $G 1$ vs $G 2$, ****Significance difference $G 1, G 2$ vs $G 3$, and ***Significance difference $G 3$ vs $G 4(p<0.001)$.

duration of trastuzumab therapy. ${ }^{4}$ Hepatitis is a common global health condition characterized by liver inflammation due to induced oxidative stress and membrane lipid peroxidation, which are the cornerstone mechanisms of this problem. ${ }^{27}$ Hence, there is a need to avoid hepatitis by using natural products such as garlic, which effectively prevent many diseases, including hepatitis. ${ }^{28,29}$

In the current study, we used an experimental model of female rats to handle easily and avoid age or sex variations. This model allows us to evaluate the effects of garlic 


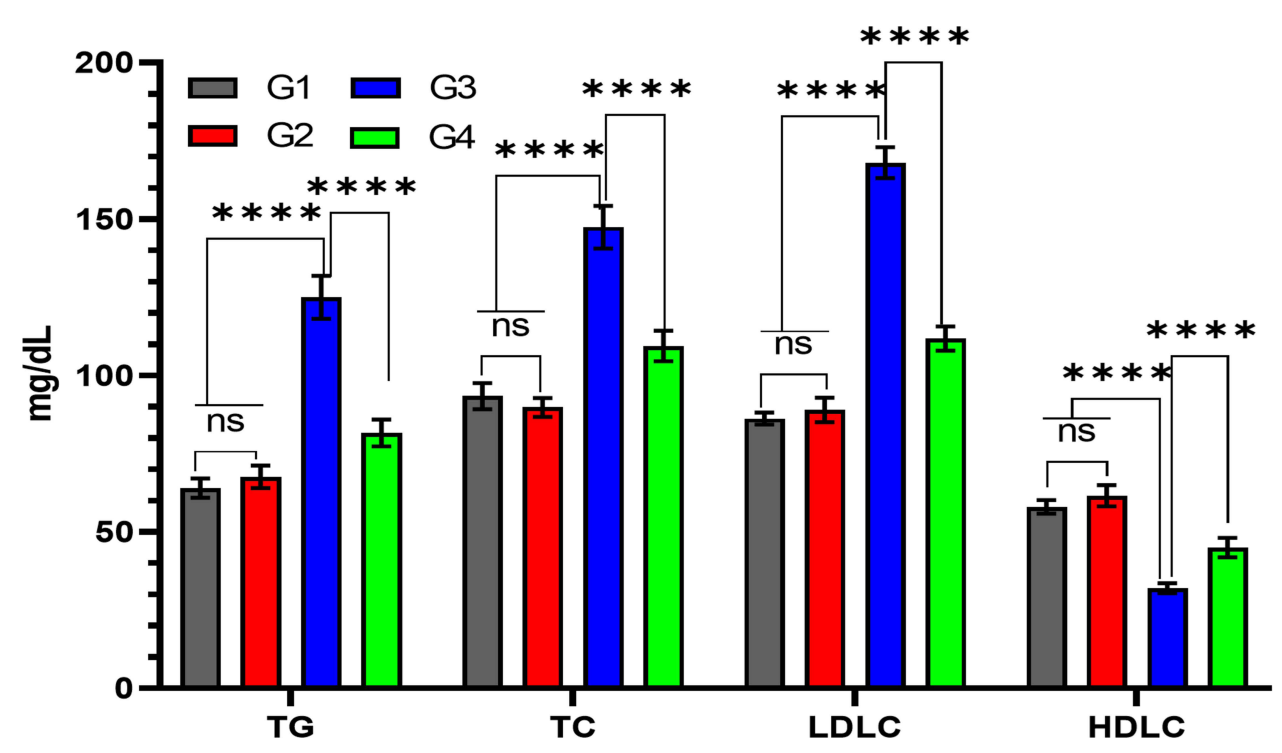

Figure 7 Quantitative analysis of garlic extract effects on trastuzumab-induced changes in the lipid profile serum levels. The levels of triglycerides (TG), total cholesterol (TC), low-density lipoprotein cholesterol (LDLC), and high-density lipoprotein cholesterol (HDLC) increase significantly in the serum of G3 compared to GI, G2, and G4. ${ }^{n}$ No significance between GI vs G2, *****Significance difference GI, G2 vs $G 3$, and G3 vs $G 4(p<0.001)$.

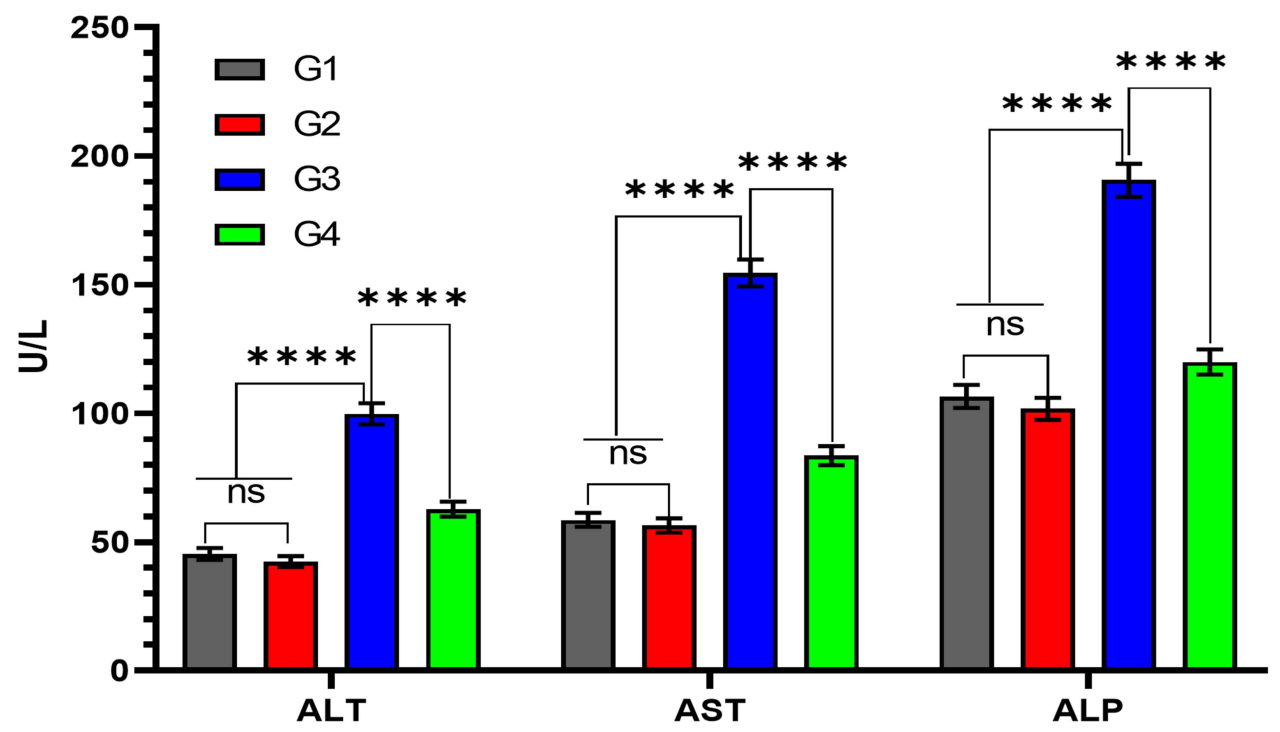

Figure 8 Quantitative analysis of garlic extract effects on trastuzumab-induced liver enzymes changes. The serum levels of alanine aminotransferase (ALT), aspartate aminotransferase (AST), and alkaline phosphatase (ALP) increase significantly in the serum of G3 compared to GI, G2, and G4. ${ }^{\text {ns }}$ No significance between GI vs G2, *****significance difference $G I, G 2$ vs $G 3$, and $G 3$ vs $G 4(p<0.00 I)$.

and understand its underlying pharmacodynamic mechanisms against trastuzumab-induced hepatotoxicity, which remain elusive. ${ }^{30}$ Herein, trastuzumab (10 $\mathrm{mg} / \mathrm{kg} /$ week) was given to rats of G3 (trastuzumab-treated rats) to induce hepatotoxicity, while garlic (500 mg/kg/day) was given to evaluate its antioxidant and anti-inflammatory effects in rats of G4 (trastuzumab+garlic-treated rats). ${ }^{14}$ Trastuzumab exhibits apparent HP diversities in the hepatic structure of G3 rats, including hepatic congestion, inflammation, fibrosis, and apoptosis compared to G1 and G2 rats. The quantitative morphometric analysis revealed significant increases in the ICs and PCNA positive cells numbers, as well as the area percentage of $\mathrm{CF}$ and TNF- $\alpha$ IE (indicators of hepatitis) in the hepatic tissues of G3 rats, indicating the enhancement of hepatic oxidative stress, inflammation, and apoptosis following trastuzumab administration. Furthermore, the quantitative analysis of biochemical measurements confirmed the HP, IHC, and morphometric results. It revealed significant low levels of SOD2, CAT, GPX1, and HDLC with significant 


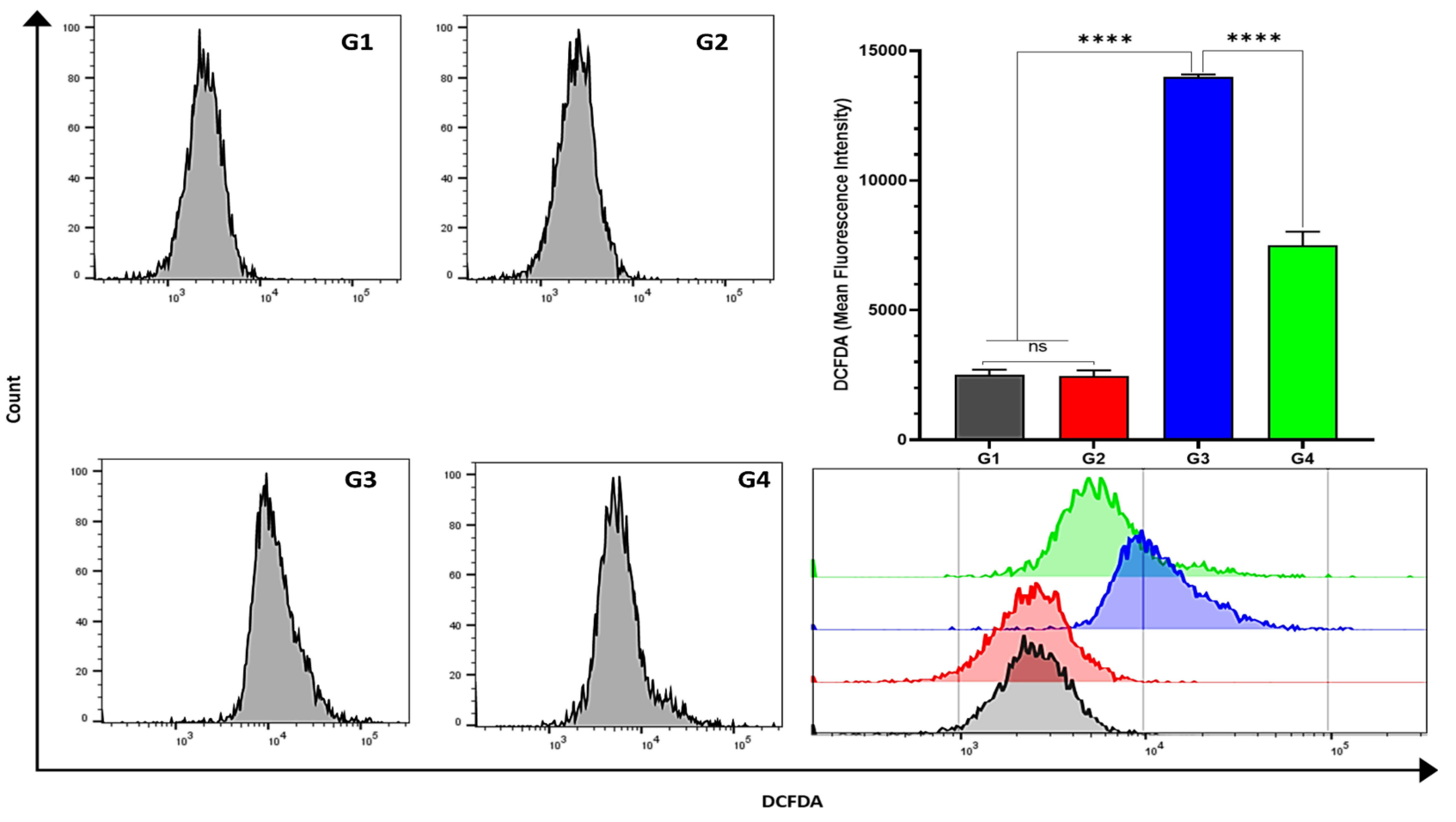

Figure 9 Quantitative analysis of garlic extract effects on Trastuzumab-induced ROS changes in the liver tissues by flow cytometry. The levels of $2^{\prime}, 7^{\prime}$-dichlorofluorescein diacetate (DCFDA) cellular reactive oxygen species (ROS) significantly increase in G3 [ 14433.3 mean fluorescent index (MFI)] and significantly decrease (7I26.5 MFI) in G4 after garlic administration. ${ }^{\text {ns }}$ No significance between GI vs G2, *****Significance difference GI, G2 vs G3, and G3 vs G4 ( $p<0.001$ ).

elevations in the serum levels of lipid profile (TG, TC, and LDLC), liver enzymes (ALT, AST, and ALP), and cellular ROS in G3 compared to G1 and G2 rats. The findings of G3 are supported by a recent study, which has endorsed significant changes in the structure of hepatocytes and disturbing the levels of hyperlipidemic status and liver enzymes (as crucial indicators of hepatocyte damage) in rats treated with trastuzumab. ${ }^{31}$

Although the underlying mechanisms of induced hepatitis in trastuzumab-treated animals have not yet been sufficiently elucidated, disruption of the HER2 signaling cascade is thought to play a role in trastuzumab-induced hepatotoxicity. In such circumstances, the actual mechanisms of trastuzumab-induced hepatitis could be explained by numerous reports, which involve enhancing oxidative stress, lipid peroxidation, overproduction of free radicals, and inflammation of hepatocytes through the induction of cell membrane injury and endorsement of hepatotoxicity. ${ }^{32-34}$ Indeed, the normal metabolism of fat prevents oxidative stress of the liver and provides ATP to the body; however, intake of trastuzumab can initiate disorders of fatty acid metabolism and induce liver injury. ${ }^{35}$ Furthermore, other studies attributed the features of hepatotoxicity (ICI, hepatic fibrosis, and apoptosis) to the expression of proinflammatory cytokines such as TNF- $\alpha$, which plays a vital role in the development of hepatotoxicity and elevates the levels of produced hepatic enzymes (ALT, AST, and ALP) above the average ranges. Trastuzumab exerts its destructive effects on hepatocytes by enhancing free radicals production, which destroys the lipids and proteins of cell membranes, impairs their cell functions, and causes cell death of hepatocytes. ${ }^{36,37}$ In addition, the tumor suppressor proteins ChK2 and P53 can regulate the mitotic activity of hepatocytes. ChK2 is a novel nuclear DNA biomarker that can arrest the cell cycle in prophase by activating the tumor suppressor protein $\mathrm{p} 53$, enhancing the pro-apoptotic caspase-3, disorganizing the centrosomes, and inducing apoptosis. ${ }^{38} \mathrm{ChK} 2$ exerts various functions such as regulating cell-cycle checkpoints or apoptosis through the p53 dependent or independent mechanism. ${ }^{39,40}$ Our data revealed reduced nuclear expression of $\mathrm{ChK} 2$ following trastuzumab induced stress through p53 independent signaling pathway as no change was observed in p53 in any treated groups (Figure 5).

Comparatively, multiple studies have proven that antiinflammatory agents can decrease the levels of inflammatory cytokines. ${ }^{41,42}$ Therefore, treatment with garlic is 
expected to reduce the exacerbations of hepatitis and improve the quality of patient's life in numerous inflammatory diseases. ${ }^{43}$ Garlic is a natural product with antioxidant, anti-inflammatory, anti-apoptotic, and antimicrobial properties. Hence, garlic can reduce chronic inflammatory diseases by modulating their inflammatory and apoptotic signaling pathways. ${ }^{44}$

Herein, our findings in rats of G4 exhibited remarkable improvements in the structure of affected hepatocytes towards the typical architecture of liver tissues (displays the classical hepatic lobules with central veins and peripheral portal tracts), with significant $(P<0.001)$ reductions in the numbers of ICs and PCNA cells, as well as the area percentage of $\mathrm{CF}$, and TNF- $\alpha$ IE. According to recent studies, these findings of liver injury could be attributed to garlic supplementation, which has numerous active components such as diallyl sulfide, allicin, ajoene, and allium. These components play a significant role against the induced oxidative stress, lipid peroxidation, inflammatory responses, and apoptotic effects of trastuzumab. $^{23,45}$ Indeed, the substantial reductions in the serum levels of lipid profile and liver enzymes in G4 rats indicated the beneficial ameliorative antiinflammatory and anti-apoptotic effects of garlic on hepatocytes of G4 compared to G3. Thereby, the significant decline in the serum levels of lipid profile tests (TG, TC, LDLC), liver function tests (ALT, AST, and ALP), and DCFDA cellular levels of ROS towards the normal levels revealed the apparent effects of garlic administration, which proved the protective antioxidant and antiinflammatory effects of garlic on hepatocytes of G4 compared to G3.

Collectively, the involved hepatoprotective mechanisms of garlic in trastuzumab-treated rats have been supported by several studies, including remarkable reductions in the production of proinflammatory mediators (suppressing the expression of proinflammatory cytokines such as TNF- $\alpha$ and nuclear factor-KB). ${ }^{46,47}$ Other recent studies reported that garlic could improve the liver function tests (ALT, AST, and ALP) through normalizing their secretions and upturning the HDLC serum levels towards the average values in numerous diseases, which provide a crucial indicator of its antioxidant and anti-inflammatory properties. $^{15,48}$ Overall, the current results proved that garlic supplementation plays a vital role as a hepatoprotective agent against trastuzumab-induced hepatitis through its antioxidant, anti-inflammatory, and anti-apoptotic effects.

\section{Conclusions}

To our knowledge, this is the first study that explores the role of garlic in alleviating trastuzumab-induced hepatotoxicity. The current findings demonstrated the hepatoprotective effect of garlic in drug-induced toxicity in detail. The study suggested that garlic extract supplementation in treating breast or gastric cancer by trastuzumab can augment its efficacy with minimal toxicity. The current study also paves the way for future research strategies using garlic and other dietary products as supplements to improve the therapeutic efficacy of chemotherapeutic drugs against various types of cancer.

\section{Data Sharing Statement}

All relevant data have been provided in the manuscript.

\section{Funding}

The authors gratefully acknowledge Qassim University, represented by the Deanship of Scientific Research, on the financial support for this research under the number [mduc-2019-2-2-I-5610] during the academic year 1440 $\mathrm{AH} / 2019$ AD.

\section{Disclosure}

The authors declare no conflicts of interest in this study. The funder had no role in the design, data collection, analyses, or interpretation of the study, writing the manuscript, or publishing the results.

\section{References}

1. Roukos DH. Trastuzumab and beyond: sequencing cancer genomes and predicting molecular networks. Pharmacogenomics J. 2011;11 (2):81-92. doi:10.1038/tpj.2010.81

2. Iqbal N, Iqbal N. Human epidermal growth factor receptor 2 (HER2) in cancers: overexpression and therapeutic implications. Mol Biol Int. 2014;2014:1-9. doi:10.1155/2014/852748

3. Arteaga CL, Sliwkowski MX, Osborne CK, Perez EA, Puglisi F, Gianni L. Treatment of HER2-positive breast cancer: current status and future perspectives. Nat Rev Clin Oncol. 2012;9(1):16. doi:10.1038/nrclinonc.2011.177

4. Ishizuna K, Ninomiya J, Ogawa T, Tsuji E. Hepatotoxicity induced by trastuzumab used for breast cancer adjuvant therapy: a case report. J Med Case Rep. 2014;8(1):1-4. doi:10.1186/1752-1947-8-417

5. Park JS, Youn J-C, Shim CY, et al. Cardiotoxicity of trastuzumab in patients with HER2-positive gastric cancer. Oncotarget. 2017;8 (37):61837. doi:10.18632/oncotarget. 18700

6. Koroglu OF, Gunata M, Vardi N, et al. Protective effects of naringin on valproic acid-induced hepatotoxicity in rats. Tissue Cell. 2021;72:101526. doi:10.1016/j.tice.2021.101526

7. Ricart AD. Drug-induced liver injury in oncology. Ann Oncol. 2017;28 (8):2013-2020. doi:10.1093/annonc/mdx158

8. Christenson ES, James T, Agrawal V, Park BH. Use of biomarkers for the assessment of chemotherapy-induced cardiac toxicity. Clin Biochem. 2015;48(4-5):223-235. doi:10.1016/j.clinbiochem.2014.10. 013 
9. Madonna R, Cadeddu C, Deidda M, et al. Improving the preclinical models for the study of chemotherapy-induced cardiotoxicity: a Position Paper of the Italian Working Group on Drug Cardiotoxicity and Cardioprotection. Heart Fail Rev. 2015;20 (5):621-631. doi:10.1007/s10741-015-9497-4

10. Rahman MM, Fazlic V, Saad NW. Antioxidant properties of raw garlic (Allium sativum) extract. Int Food Res J. 2012;19 (2):589-591.

11. Khan A, Shukla Y, Kalra N, et al. Potential of diallyl sulfide bearing $\mathrm{pH}$-sensitive liposomes in chemoprevention against DMBA-induced skin papilloma. Mol Med. 2007;13(7-8):443-451. doi:10.2119/200600111.Khan

12. Abed HH, Yakoob AY. Study the protective and therapeutic effects of crude garlic on mortality, oocyst output and hepatic lesions in experimental infection with Eimeria stiedae in domestic rabbits. Bas $J$ Vet Res. 2013;12(2):314-331. doi:10.33762/bvetr.2013.83827

13. Tandon SK, Singh S, Prasad S. Influence of garlic on the disposition and toxicity of lead and cadmium in the rat. Pharm Biol. 2001;39 (6):450-454. doi:10.1076/phbi.39.6.450.5887

14. Thomson M, Al-Qattan KK, Divya JS, Ali M. Anti-diabetic and anti-oxidant potential of aged garlic extract (AGE) in streptozotocin-induced diabetic rats. BMC Complement Altern Med. 2015;16(1):1-9. doi:10.1186/s12906-016-0992-5

15. Pal R, Vaiphei K, Arbab Sikander KS, Rana ZT. Effect of garlic on isoniazid and rifampicin-induced hepatic injury in rats. World $J$ Gastroenterol. 2006;12(4):636. doi:10.3748/wjg.v12.i4.636

16. Nicol M, Sadoune M, Azibani F, et al. Effect of metoprolol in a chronic murine model of cardiotoxicity induced by doxorubicin and trastuzumab. Arch Cardiovasc Dis Suppl. 2019;11(1):109. doi:10.1016/j.acvdsp.2018.10.241

17. Eryılmaz A, Demirci B, Günel C, et al. Evaluation of lapatinib and trastuzumab for ototoxic effects. $J$ Int Adv Otol. 2015;11(3):207.

18. Chang H-Y, Morrow K, Bonacquisti E, Zhang W, Shah DK. Antibody pharmacokinetics in rat brain determined using microdialysis. In: MAbs. Vol. 10. Taylor \& Francis; 2018:843-853.

19. Feldman AT, Wolfe D. Tissue processing and hematoxylin and eosin staining. In: Histopathology. Springer; 2014:31-43.

20. Mousa AM, El-Mahalaway AM, El-Desoky RE. The protective effects of sodium hyaluronate on corneal injuries induced by chlorinated water in adult male rabbits: a histological and immunohistochemical study. Egypt J Histol. 2018;41(2):140-149. doi:10.21608/ EJH.2018.13835

21. Hussien NI, Mousa AM. Could nitric oxide be a mediator of action of oxytocin on myocardial injury in rats? (Biochemical, histological and immunohistochemical study). Gen Physiol Biophys. 2016;35 (3):353-362. doi:10.4149/gpb_2015049

22. Ye F, Xie DX, Lu YP, Gao QL. Silencing of cell cycle checkpoint kinase gene enhances cisplatin-induced apoptosis of lung cancer cells. Zhonghua Zhong Liu Za Zhi [Chin J Oncol]. 2009;31 (11):804-809.

23. Khan A, Alhumaydhi FA, Alwashmi ASS, et al. Diallyl sulfide-mediated modulation of the Fatty Acid Synthase (FASN) leads to cancer cell death in BaP-induced lung carcinogenesis in Swiss mice. J Inflamm Res. 2020;13:1075. doi:10.2147/JIR.S28 4279

24. Srinivasan S, Parsa V, Liu CY, Fontana JA. Trastuzumab-induced hepatotoxicity. Ann Pharmacother. 2008;42(10):1497-1501. doi:10. 1345/aph.1L217

25. Mousa AM, Aldebasi YH. L-carnosine mitigates interleukin-1 $\alpha$ induced dry eye disease in rabbits via its antioxidant, antiinflammatory, antiapoptotic, and antifibrotic effects. Cutan Ocul Toxicol. 2021;1-31. doi:10.1080/15569527.2021.2003377

26. Viani GA, Afonso SL, Stefano EJ, de Fendi LI, Soares FV. Adjuvant trastuzumab in the treatment of her-2-positive early breast cancer: a meta-analysis of published randomized trials. BMC Cancer. 2007;7 (1):1-11. doi:10.1186/1471-2407-7-153
27. Bomgning CLK, Sinda PVK, Ponou BK, et al. Hepatoprotective effects of extracts, fractions and compounds from the stem bark of Pentaclethra macrophylla Benth: evidence from in vitro and in vivo studies. Biomed Pharmacother. 2021;136:111242. doi:10.1016/j. biopha.2021.111242

28. Guan M-J, Zhao N, Xie K-Q, Zeng T. Hepatoprotective effects of garlic against ethanol-induced liver injury: a mini-review. Food Chem Toxicol. 2018;111:467-473. doi:10.1016/j.fct.2017.11.059

29. Lee HS, Lim WC, Lee SJ, et al. Hepatoprotective effects of lactic acid-fermented garlic extract against Acetaminophen-induced acute liver injury in rats. Food Sci Biotechnol. 2016;25(3):867-873. doi:10.1007/s10068-016-0143-2

30. Vucicevic D, Carey EJ, Karlin NJ. Trastuzumab-induced hepatotoxicity: a case report. Breast Care. 2013;8(2):146-148. doi:10.1159/ 000346844

31. Mohan N, Shen Y, Endo Y, ElZarrad MK, Wu WJ. Trastuzumab, but not pertuzumab, dysregulates HER2 signaling to mediate inhibition of autophagy and increase in reactive oxygen species production in human cardiomyocytes. Mol Cancer Ther. 2016;15(6):1321-1331. doi:10.1158/1535-7163.MCT-15-0741

32. Paracha UZ, Fatima K, Alqahtani $\mathrm{M}$, et al. Oxidative stress and hepatitis C virus. Virol J. 2013;10(1):1-9. doi:10.1186/1743-422X10-251

33. Aydin BK, Sarikaya H, Tanyel E, Esen S, Sunbul M, Leblebicioglu $\mathrm{H}$. Oxidative stress and antioxidant defense in patients with chronic hepatitis B. Clin Lab. 2012;58(3-4):273-280.

34. Muriel P. Role of free radicals in liver diseases. Hepatol Int. 2009;3 (4):526-536. doi:10.1007/s12072-009-9158-6

35. Zhuang T, Gu X, Zhou N, Ding L, Yang L, Zhou M. Hepatoprotection and hepatotoxicity of Chinese herb Rhubarb (Dahuang): how to properly control the "General (Jiang Jun)" in Chinese medical herb. Biomed Pharmacother. 2020;127:110224. doi:10.1016/j.biopha.2020.110224

36. Baudi I, Isogawa M, Moalli $\mathrm{F}$, et al. Interferon signaling suppresses the unfolded protein response and induces cell death in hepatocytes accumulating hepatitis B surface antigen. PLoS Pathog. 2021;17(5): e1009228. doi:10.1371/journal.ppat.1009228

37. Sharifudin SA, Fakurazi S, Hidayat MT, Hairuszah I, Aris Mohd Moklas M, Arulselvan P. Therapeutic potential of Moringa oleifera extracts against Acetaminophen-induced hepatotoxicity in rats. Pharm Biol. 2013;51(3):279-288. doi:10.3109/13880209.2012.72 0993

38. Castedo M, Perfettini J-L, Roumier T, et al. The cell cycle checkpoint kinase Chk2 is a negative regulator of mitotic catastrophe. Oncogene. 2004;23(25):4353-4361. doi:10.1038/sj.onc.1207573

39. van Jaarsveld MTM, Deng D, Ordoñez-Rueda D, Paulsen M, Wiemer EAC, Zi Z. Cell-type-specific role of CHK2 in mediating DNA damage-induced G2 cell cycle arrest. Oncogenesis. 2020;9 (3):35. doi:10.1038/s41389-020-0219-y

40. Aliouat-Denis CM, Dendouga N, Van den Wyngaert I, et al. p53independent regulation of $\mathrm{p} 21 \mathrm{Waf} 1 / \mathrm{Cip} 1$ expression and senescence by Chk2. Mol Cancer Res. 2005;3(11):627-634. doi:10.1158/15417786.MCR-05-0121

41. Alrumaihi F. Garlic and its active compounds: a novel strategy to fight diseases through modulating biological activities. Pharmacogn J. 2020;12(6):1463-1474. doi:10.5530/pj.2020.12.201

42. Wilson EA, Demmig-Adams B. Antioxidant, anti-inflammatory, and antimicrobial properties of garlic and onions. Nutr Food Sci. 2007;37 (3):178-183. doi:10.1108/00346650710749071

43. Tran G-B, Dam S-M, Le N-T-T. Amelioration of single clove black garlic aqueous extract on dyslipidemia and hepatitis in chronic carbon tetrachloride intoxicated Swiss Albino mice. Int $J$ Hepatol. 2018;2018:1-9. doi:10.1155/2018/9383950

44. El-Saber Batiha G, Magdy Beshbishy A, G Wasef L, et al. Chemical constituents and pharmacological activities of garlic (Allium sativum L.): a review. Nutrients. 2020;12(3):872. doi:10.3390/nu12030872 
45. Shang A, Cao S-Y, Xu X-Y, et al. Bioactive compounds and biological functions of garlic (Allium sativum L.). Foods. 2019;8(7):246. doi:10.3390/foods 8070246

46. Dehghani S, Alipoor E, Salimzadeh A, et al. The effect of a garlic supplement on the pro-inflammatory adipocytokines, resistin and tumor necrosis factor-alpha, and on pain severity, in overweight or obese women with knee osteoarthritis. Phytomedicine. 2018;48:70-75. doi:10.1016/j.phymed.2018.04. 060
47. Ziamajidi N, Nasiri A, Abbasalipourkabir R, Sadeghi Moheb S. Effects of garlic extract on TNF- $\alpha$ expression and oxidative stress status in the kidneys of rats with STZ+ nicotinamide-induced diabetes. Pharm Biol. 2017;55(1):526-531. doi:10.1080/13880209.2016.1255978

48. Panjeshahin A, Mollahosseini M, Panbehkar-Jouybari M, Kaviani M, Mirzavandi F, Hosseinzadeh M. Effects of garlic supplementation on liver enzymes: a systematic review and meta-analysis of randomized controlled trials. Phytother Res. 2020;34(8):1947-1955. doi:10.1002/ ptr.6659

\section{Publish your work in this journal}

The Journal of Inflammation Research is an international, peerreviewed open-access journal that welcomes laboratory and clinical findings on the molecular basis, cell biology and pharmacology of inflammation including original research, reviews, symposium reports, hypothesis formation and commentaries on: acute/chronic inflammation; mediators of inflammation; cellular processes; molecular mechanisms; pharmacology and novel anti-inflammatory drugs; clinical conditions involving inflammation. The manuscript management system is completely online and includes a very quick and fair peerreview system. Visit http://www.dovepress.com/testimonials.php to read real quotes from published authors. 